\title{
Thermal development of a calixarene resist
}

\author{
V. Auzelyte, ${ }^{\text {a) }}$ A. Langner, and H. H. Solak \\ Laboratory for Micro- and Nanotechnology, Paul Scherrer Institut, 5232 Villigen PSI, Switzerland
}

(Received 15 July 2009; accepted 24 August 2009; published 4 December 2009)

\begin{abstract}
Thermal development of photoresist films in lithography is an interesting alternative to the common wet development technique, which can result in problems such as swelling, line edge roughness, and pattern collapse. The authors investigated thermal development of a nonchemically amplified calixarene type high-resolution electron beam resist. Isolated and dense structures were developed at $250-400{ }^{\circ} \mathrm{C}$ in air and in low vacuum conditions. They achieved $25 \mathrm{~nm}$ half-pitch dense line/ space patterns and $17 \mathrm{~nm}$ isolated lines without sacrificing resist resolution, sensitivity, or contrast. The obtained lines were smoother with more than two times smaller line edge roughness. The results demonstrate the feasibility of using thermal development for high-resolution nanolithography.

(C) 2009 American Vacuum Society. [DOI: 10.1116/1.3237137]
\end{abstract}

Development is one of the critical steps in a lithographic process that gives the micro- or nanostructures in the resist their final shape. In this step, depending on whether the resist is positive or negative tone, the exposed or unexposed parts of the resist are removed from the substrate surface. Besides the conventional wet development process, there are a number of different development approaches. One of them, first proposed more than two decades ago, is the thermal development technique. ${ }^{1}$ During the lithographic exposure process or subsequent modification steps such as the postexposure bake, the resist film is chemically modified. This modification often means cross-linking or depolymerisation of the resist. Thermal development takes advantage of the difference between volatility of these two resist states. Upon thermal treatment, resist regions that consist of lighter molecules become volatile and leave the surface while regions with larger or cross-linked molecules remain to form the desired pattern in the resist.

Dry resist development techniques attract interest as they are expected to have a number of advantages over the prevalent wet development processes. First of all, the absence of hazardous solvents makes them more environmentally friendly and potentially cheaper. In addition, some of the notorious problems that are inherent to the conventional process such as resist swelling, increased line edge roughness (LER), capillary-force driven resist collapse, and pattern detachment from the substrate may be avoided through the use of dry development techniques.

The principle of thermal development was first demonstrated for polymer resists. ${ }^{1}$ Thermal development is a relatively low temperature process, typically requiring temperatures in the $100-300^{\circ} \mathrm{C}$ range. In some of the early studies, introduction of chemical amplification helped to reduce the development temperatures from above $200{ }^{\circ} \mathrm{C}$ to below $100{ }^{\circ} \mathrm{C}^{2,3}$ In these studies thermal development was also shown to improve resist sensitivity and no resist flow was observed. More recently, resist systems dedicated and optimized for a lower development temperature and clean de-

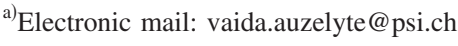

composition were developed. ${ }^{4,5}$ In a related approach heat treatment applied after wet development was shown to shrink structures and improve LER. ${ }^{6}$ Fully developed submicrometer structures were made using UV and electron beam exposures of poly(4-chlorophthalaldehyde). ${ }^{3}$ Further approaches include thermal nanolithography with a molecular resist using atomic force microscopy tip to write a pattern with development at $105^{\circ} \mathrm{C}$ to give lines with widths down to $45 \mathrm{~nm},{ }^{7}$ and demonstration of monomolecular patterns using the thermal desorption of pristine self-assembled monolayers. ${ }^{8}$

Organic molecular resists are good candidates for thermal development because of their small molecular size, which facilitates removal from substrate and enables highresolution patterning. For this reason we chose a calixarenetype resist, $p$-chloromethyl-metoxy-calix[4]arene (TEBN-1, Tokuyama Corp., Japan) and performed patterning experiments using low-voltage electron-beam exposures. Calixarene is a cyclic oligomer and has antioxidant properties and good thermal stability. In particular, TEBN-1 is a high resolution molecular electron beam resist for sub-10 nm fabrication ${ }^{9}$ and $12.5 \mathrm{~nm}$ half-pitch dense line/space patterns have been obtained in this resist using EUV interference lithography. ${ }^{10}$ In our low voltage electron beam exposures (at $2.5 \mathrm{keV}$ ), we observed typical hairlike roughness between the lines (see example in Fig. 5), when the resist is developed with isopropyl alcohol (IPA). An alternative developer is xylene, which improves the contrast at the expense of lowered sensitivity and the appearance of a significant swelling problem. ${ }^{11}$ By using a thermal development approach we were able to simplify the development and address the line edge roughness and swelling problems.

TEBN-1 resist films of $25-27 \mathrm{~nm}$ were spin coated at a speed of $3000 \mathrm{rpm}$ on silicon or chromium-coated silicon substrates. The substrates were pretreated with oxygen plasma to improve resist adhesion. The samples were baked at $110{ }^{\circ} \mathrm{C}$ for 1 min after coating. Box patterns for contrast evaluation and isolated and dense line patterns with 25-100 $\mathrm{nm}$ half pitches were exposed with a $2.5 \mathrm{keV}$ electron beam in a LION LV1 system. Thermal development of the exposed 


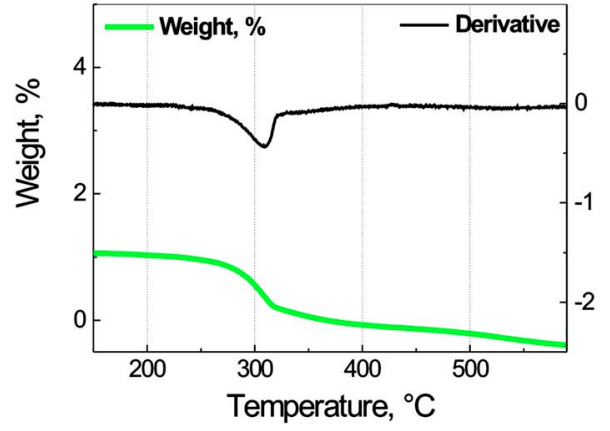

FIG. 1. (Color online) Plot showing the results of the thermo gravimetric analysis (TGA) of unexposed TEBN-1 calixarene.

samples was carried out at $200-550{ }^{\circ} \mathrm{C}$ by placing the samples on a hot plate in air or in a low vacuum at 0.1 mbar. For comparison with wet processing some of the samples were developed in IPA for $30 \mathrm{~s}$.

When choosing the development temperature it was necessary to ensure that certain conditions were met. First, a dramatically different volatility difference between unexposed and exposed materials is required. Second, at a specific temperature the resist should become volatile or degrade into volatile products rather then oxidizing and/or cross-linking and remaining on the surface. Finally, resist melting or flow should be minimal. It is possible to gain an idea of the temperature at which optimum volatility difference between exposed and unexposed materials occurs using thermogravimetric analysis (TGA). A TGA curve of unexposed TEBN-1 resist has been recorded and is shown in Fig. 1. This test was performed by drying a droplet of the resist in a small metal container that is normally used in the TGA tool. The main resist mass-loss step occurs at about $300{ }^{\circ} \mathrm{C}$. After heating to $500{ }^{\circ} \mathrm{C}$ a carbon-black layer formed in the TGA holder, pointing to the possible creation of residual products by the thermal treatment. However, this layer was not observed on spin-coated thin-film samples which were treated in the same temperature range. It is possible that the large amount of material that was used in the TGA test was responsible for the visible residue. TGA tests were not possible on the exposed resist due to the need to expose large amounts of the material. Therefore, the main information that is gathered from the TGA test is the need to go above about $300{ }^{\circ} \mathrm{C}$ to render the unexposed resist volatile. However, the removal of thin TEBN-1 films was visually observed at temperatures above $210{ }^{\circ} \mathrm{C}$ even though no significant mass loss was seen at this temperature in the TGA test. Also, in thermal development tests we observed that above about $520^{\circ} \mathrm{C}$, all material, exposed and unexposed, is instantly removed from the surface.

The contrast and sensitivity of the process for the samples developed in the $250-400{ }^{\circ} \mathrm{C}$ range for $15 \mathrm{~s}$ are shown in Fig. 2. The contrast $\gamma$ is defined as $\log \left(D_{1} / D_{0}\right)^{-1}$, where $D_{0}$ is the dose that starts to cross-link the resist and $D_{1}$ is the dose at $80 \%$ of the cross-linked resist thickness. The measurements were performed on exposed large box fields with a mechanical profilometer. The contrast obtained in thermal

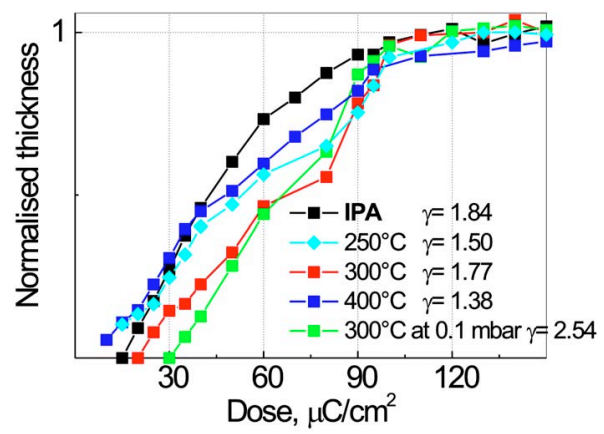

FIG. 2. (Color online) Contrast curves of calixarene developed thermally on a hot plate at 250,300 , and $400{ }^{\circ} \mathrm{C}$ for $15 \mathrm{~s}$ in air and $300{ }^{\circ} \mathrm{C}$ at $0.1 \mathrm{mbar}$ for $660 \mathrm{~s}$ in comparison to standard development in IPA.

development was optimal at $300{ }^{\circ} \mathrm{C}(\gamma=1.77)$ and is comparable to the standard IPA process $(\gamma=1.84)$. The sensitivity is practically the same as the IPA-development process. At higher temperatures the contrast decreases probably due to resist melting or effects such as oxidation or other temperature induced chemical resist modifications. Development in low vacuum helped to improve the contrast from 1.77 to 2.54. This change is possibly due to much lower concentrations of $\mathrm{O}_{2}$ and water vapor that may cause contrast reducing chemical reactions in the resist.

The curves shown in Fig. 2 are normalized, but in practice the actual amount of resist left on the substrate surface is important for subsequent pattern-transfer processes. Excessive loss of resist thickness in exposed areas (i.e., top loss) is an undesired property. Remaining resist thickness after development is shown in Fig. 3 as a function of development temperature. Only $6 \%$ of the thickness is lost at $250{ }^{\circ} \mathrm{C}$ and $12 \%$ at $300{ }^{\circ} \mathrm{C}$. Developing at higher temperature results in more thickness loss, such as $38 \%$ at $500{ }^{\circ} \mathrm{C}$, possibly due to the loss of partially cross-linked molecules in the resist and higher vaporization rate of cross-linked molecules at this temperature. At lower temperatures $\left(250-300{ }^{\circ} \mathrm{C}\right)$ the small thickness loss may be due to ability of molecules that are not cross-linked at all to diffuse out and leave the film.

Isolated and $30 \mathrm{~nm}$ half pitch dense lines developed in both ways, on a hot plate at $300{ }^{\circ} \mathrm{C}$ for $15 \mathrm{~s}$ in air and in IPA for $30 \mathrm{~s}$, are shown in Figs. 4(a)-4(d). The grainy texture of

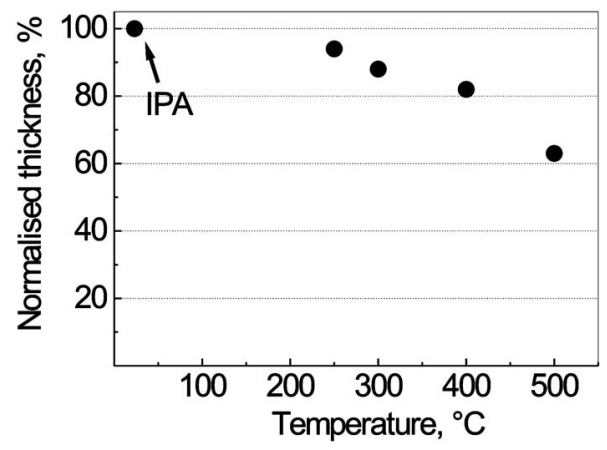

FIG. 3. Thickness of electron beam exposed calixarene developed in conventional IPA developer and thermally at $250-500{ }^{\circ} \mathrm{C}$. 


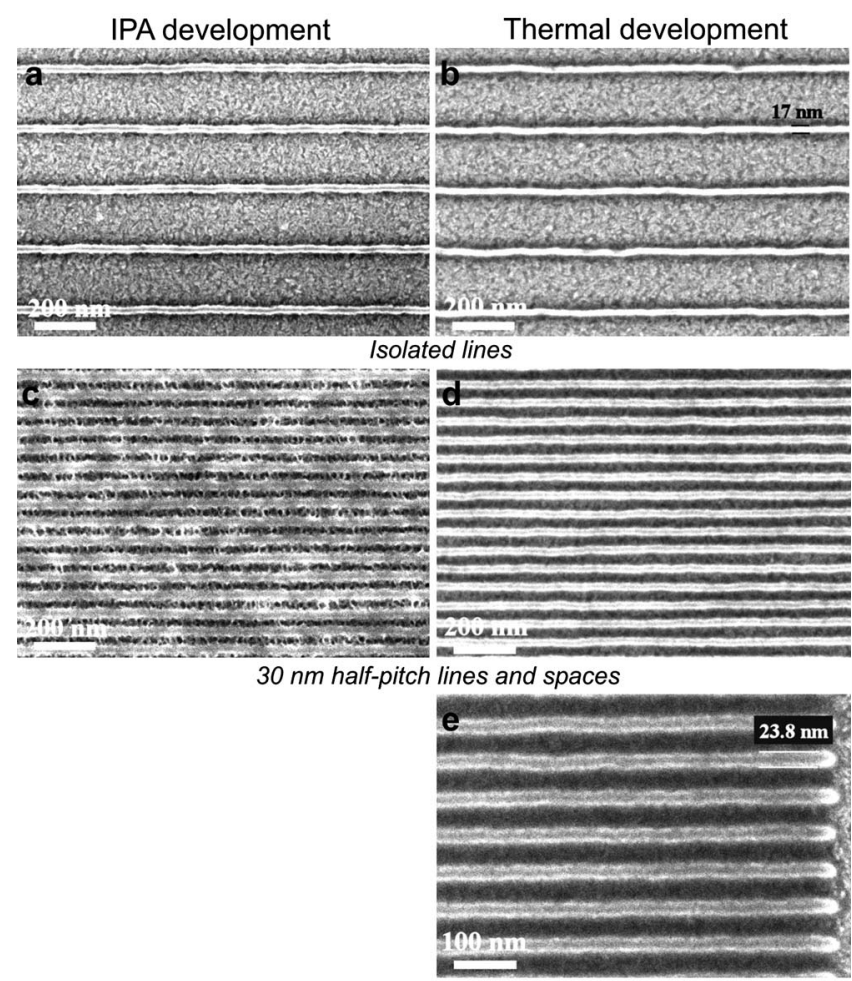

$25 \mathrm{~nm}$ half-pitch lines and spaces

FIG. 4. Isolated and dense line patterns written with electron beam. [(a) and (c)] Developed in IPA for $30 \mathrm{~s}$ and [(b)-(e)] developed thermally at $300{ }^{\circ} \mathrm{C}$ for $15 \mathrm{~s}$.

chromium which is visible between the lines shows that both $100 \mathrm{~nm}$ period isolated structures are fully developed and have a good line definition. One of the main advantages of thermal development is apparent in the comparison of the dense structures. A typical dense line pattern with $30 \mathrm{~nm}$ half pitch seen in Fig. 4(c) obtained after IPA development has hairlike lines with line edge roughness of $9.6 \mathrm{~nm}$. Figure 4(d) shows much improved pattern thermally developed at $300{ }^{\circ} \mathrm{C}$ with smoother lines and line edge roughness of only $3.9 \mathrm{~nm}$ (analyzed with software package LWR DEMOKRITOS). The smallest $25 \mathrm{~nm}$ half-pitch line pattern is shown in Fig. 4(e).

In thermally developed large area patterns, we noticed the presence of a residual film between the lines and around the patterned area. A similar phenomenon was also observed in the exposure of $20 \times 20 \mu \mathrm{m}^{2}$ boxes made for contrast measurement. The boxes were developed in IPA for $30 \mathrm{~s}$ [Fig. 5 (a)] and at $400{ }^{\circ} \mathrm{C}$ for $15 \mathrm{~s}$ in air [Fig. 5(b)], respectively. At low doses the residual film forms a circular shape, which is likely to be due to the diffusion of partially cross-linked molecules, which are able to diffuse on the substrate but are not able to leave the surface. At higher doses clear square-shaped boxes are formed. The contrast curves were built using clearly developed parts of the boxes avoiding the flowing resist. This problem is similar to the residual film that is always present in patterns created with nanoimprint lithogra-
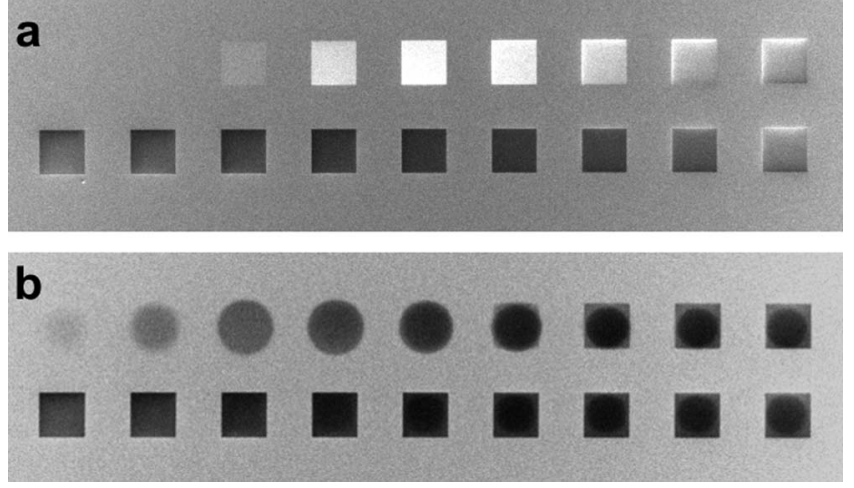

FIG. 5. $20 \times 20 \mu \mathrm{m}^{2}$ box patterns developed (a) in IPA for $30 \mathrm{~s}$ and (b) thermally at $400{ }^{\circ} \mathrm{C}$ for $15 \mathrm{~s}$. The dose is increasing in the top row from left to right, in the bottom row from the right to the left, with the step of $10 \mu \mathrm{C} / \mathrm{cm}^{2}$.

phy (NIL). Therefore, the solution that is universally applied in NIL, i.e., reactive ion etching of the residual film, may also be applied to thermally developed resists.

In conclusion, we have demonstrated thermal development of a nonchemically amplified molecular calixarene resist. Calixarene resist developed at $300{ }^{\circ} \mathrm{C}$ on a hot plate maintains the sensitivity, contrast, and good resolution properties. The obtained resolution of $17 \mathrm{~nm}$ for isolated lines and $25 \mathrm{~nm}$ half-pitch dense patterns proves the competitiveness of this process with other advanced nanolithography methods. The technique may be useful as a remedy for the notorious LER problem that is faced in nanolithography. Future improvements for the thermal development process include a reduction in the high development temperature by optimization of the resist chemistry and future optimization of the vacuum conditions to provide an optimal pattern.

The authors gratefully acknowledge Dr. H. Ben Youcef from PSI and M. Colussi from ETHZ for the help with TGA measurements.

${ }^{1}$ F. M. Houlihan, F. Bouchard, J. M. J. Frechet, and C. G. Willson, Macromolecules $\mathbf{1 9}, 13$ (1986).

${ }^{2}$ J. M. J. Fréchet, F. Bouchard, E. Eichler, F. M. Houlihan, T. Iizawa, B. Kryczka, and C. G. Willson, Polym. J. (Tokyo, Jpn.) 19, 31 (1987).

${ }^{3}$ H. Ito, M. Ueda, and R. Schwalm, J. Vac. Sci. Technol. B 6, 2259 (1988).

${ }^{4}$ S. N. Ali, S. Ghafouri, Z. Yin, P. Froimowicz, S. Begum, and M. A. Winnik, Eur. Polym. J. 44, 4129 (2008).

${ }^{5}$ V. Van Speybroeck, Y. Martelé, M. Waroquier, and E. Schacht, J. Am. Chem. Soc. 123, 10650 (2001).

${ }^{6}$ S. H. Kim, H. Hiroshima, and M. Komuro, Nanotechnology 17, 2219 (2006).

${ }^{7}$ S. Noach, M. Manevich, N. P. Eisenberg, E. P. Fokin, and T. V. Mihalina, Nanotechnology 16, 775 (2005).

${ }^{8}$ A. Turchanin, M. El-Desawy, and A. Gölzhäuser, Appl. Phys. Lett. 90, 053102 (2007).

${ }^{9}$ M. Ishida, J. Fujita, T. Ogura, Y. Ohiai, E. Ohshima, and J. Momoda, Jpn. J. Appl. Phys., Part 1 42, 3913 (2003).

${ }^{10}$ H. H. Solak, Y. Ekinci, P. S. Käser, and S. Park, J. Vac. Sci. Technol. B 25, 91 (2007).

${ }^{11}$ M. Narihiro, K. Arai, M. Ishida, Y. Ochiai, and Y. Natsuka, Jpn. J. Appl. Phys., Part 1 44, 5581 (2005). 